\section{ORIGINAL RESEARCH}

Q. Miao

X.L. Zhao

Q.Y. Zhang

Z.Y. Zhang

Y.H. Guan

H.Y. Ye

S. Zhang

M.F. Zeng

C.T. Zuo

Y.M. Li

\title{
Stability in Brain Glucose Metabolism Following Brown Adipose Tissue Inactivation in Chinese Adults
}

BACKGROUND AND PURPOSE: The thermogenesis of BAT is believed to be controlled through some pathways initiated in the brain, though the changes in brain activity among different states of BAT-positive subjects are still unclear. We hypothesized that some significant differences of regional cerebral metabolism between various groups were related to the BAT activities regardless of temperature changes.

MATERIALS AND METHODS: Relative regional cerebral glucose metabolism was compared between 15 healthy subjects with activated BAT and 30 healthy controls without activated BAT by using a brain FDG-PET scan. A follow-up PET scan was performed to assess metabolic changes of the brain when BAT activity was eliminated by heat exposure.

RESULTS: Compared with controls, BAT-positive subjects exhibited lower activity in the inferior parietal lobule, limbic system, and frontal lobe and higher activity in the precuneus before heat exposure. Compared with the BAT elimination status, subjects with activated BAT showed a decreased metabolism in the parietal lobe, frontal lobe, culmen, cingulate gyrus, and sublobar region. Compared with controls, BAT-positive subjects after BAT inactivation had significant hypometabolic areas in the temporal lobe and limbic lobe and hypermetabolic areas in the parietal lobe.

CONCLUSIONS: Our findings illustrate that changes of regional cerebral metabolism are related to BAT activities regardless of temperature changes. This before-after controlled study supports the finding that the brain responses appear to be active in modulating the metabolic function of BAT activity.

ABBREVIATIONS: $\mathrm{BA}=$ Brodmann area; $\mathrm{BAT}=$ brown adipose tissue; $\mathrm{BMI}=$ body mass index; ${ }^{18} \mathrm{~F}-\mathrm{FDG}=$ fluorine $18 \mathrm{FDG} ; \mathrm{FDR}=$ false discovery rate; $\mathrm{FPG}=$ fasting plasma glucose; $\mathrm{MNI}=$ Montreal Neurological Institute; $Z_{\max }=$ maximum of $Z$ value

B AT is the major site for cold- and diet-induced thermogenesis, maintaining body temperature and energy homeostasis in small mammals and neonate humans, particularly during cold exposure without shivering. However, BAT has been considered essentially nonexistent with no physiologic relevance in adults. ${ }^{1,2}$ In 2007, Nedergaard et $\mathrm{al}^{3}$ reviewed several studies showing that BAT is present in adult humans; moreover, adult BAT activity is acutely cold-induced

Received November 1, 2011; accepted after revision December 7.

From the Division of Endocrinology and Metabolism (Q.M., X.L.Z., Q.Y.Z., Z.Y.Z., H.Y.Y., S.Z., M.F.Z., Y.M.L.), Department of Internal Medicine, and PET Center (Y.H.G.. C.T.Z.), Division of Nuclear Medicine, Department of Radiology, Huashan Hospital, Shanghai Medical College, Fudan University, Shanghai, China.

Qing Miao and Xue Lan Zhao contributed equally to this work.

This work was supported by grants from the National Natural Science Foundation of China (No. 30771024, No. 30900502, No. 30800344, and No. 81070680); Shanghai Committee of Science and Technology, China (No. 10JC1401002); Young Science Fund of Fudan University (No. 09F071); Institutes of Biomedical Sciences, Fudan University (No.2009056); and Graduate Innovation Fund of Fudan University (No. EYF151063).

Please address correspondence to ChuanTao Zuo, MD, PhD, PET Center, Division of Nuclear Medicine, Huashan Hospital, Shanghai Medical College, Fudan University, 518 Wuzhong East Rd, Shanghai 200235, China; e-mail: zuoct_cn@hotmail.com; or Yi Ming Li, MD, PhD, Division of Endocrinology and Metabolism, Huashan Hospital, Department of Internal Medicine, Shanghai Medical College, Fudan University, 12 Wulumuqi Middle Rd, Shanghai 200040, China; e-mail: yimingli.fudan@gmail.com

Indicates open access to non-subscribers at www.ajnr.org

Indicates article with supplemental on-line tables.

Indicates article with supplemental on-line figure.

http://dx.doi.org/10.3174/ajnr.A3006 and stimulated via the sympathetic nervous system. During 2009, strong evidence for the existence of active BAT in adults came from combined ${ }^{18} \mathrm{~F}$-FDG-PET/CT imaging and led to a paradigm shift in our understanding of the potential role of BAT in adult humans. ${ }^{4,5}$

The innervation of BAT by the sympathetic nervous system is incontrovertible and, with its activation, possibly an exclusive stimulator of BAT thermogenesis. ${ }^{6}$ There are more and more data supporting the importance of nervous system regulation of BAT activity. The rate of thermogenesis is believed to be controlled by some pathways initiated in the brain and to cover the primary location of coordinating and integrating thermoregulatory responses. Many studies hypothesized that the neural circuits within the central nervous system-primarily in the hypothalamus - control BAT heat production by regulating BAT sympathetic nerve activity. ${ }^{7-9}$ Although there is evidence suggesting that BAT function is affected by the nervous system in lower mammals, for humans this connection remains unclear. Recently, 1 study in 21 patients with activated BAT and 21 matched controls without activated BAT showed that BAT-positive patients had a significant hypermetabolic area in the right inferior parietal lobule (BA 40) and significant hypometabolic areas in the left insula (BA 13) and right cerebellum. ${ }^{10}$ Taken together, there is a neural feedback loop between adipose tissue and brain, which plays a major role in regulation of energy homeostasis. However, the changes in brain activity among different states of BAT-positive subjects are unclear. 
In this study, we performed ${ }^{18} \mathrm{~F}$-FDG PET/CT examinations in BAT-positive volunteers. We present the findings from cerebral glucose metabolism measurements in BAT-positive individuals before and after heat exposure. Our particular interests were the possible abnormal metabolism in specific brain regions regardless of temperature changes. We hypothesized that some changes of regional cerebral glucose metabolism are related to BAT activities and that other changes that can be reversed by heat exposure are related to temperature response.

\section{Materials and Methods}

\section{Subjects and Procedures}

Between December 2009 and May 2010, a group of 15 healthy subjects with activated BAT ( 10 women, 5 men; mean age, $35.67 \pm 6.66$ years; range, 25-46 years) were collected at the Huashan PET/CT center, Fudan University. We then searched for matched controls with inactivated BAT. We preferred to select control scans obtained during the same week as those in the activated BAT group. Then, scans and reports for 30 sex-, age-, BMI-, outdoor temperature-, and FPGmatched healthy controls without activated BAT (20 women, 10 men; mean age, $37.43 \pm 6.14$ years; range, $26-47$ years) (On-line Table 1 ) were collected. None of these subjects had received $\beta$-blockers to prevent activating BAT before the PET/CT scan. Screening procedures included complete medical examinations, routine laboratory evaluations, and MR imaging (Trio 3T; Siemens, Shanghai, China) to exclude potential participants with significant body and brain diseases, and a neuropsychiatric evaluation.

Subjects were studied in the morning, from approximately 7 to 11 AM, after an overnight fast for 8 hours. During the experiment, all subjects wore standardized clothing ( $0.79 \mathrm{clo}$; "clo" is a unit of measure for the insulating properties of clothing). The PET-CT scanning protocol (PET/CT, Biograph 64; Siemens) included confirmation of the intravenous injection of $5.55-7.40 \mathrm{MBq} / \mathrm{kg}$ of ${ }^{18} \mathrm{~F}-\mathrm{FDG}$. In a climate chamber, the subjects rested in a supine position under thermoneutral conditions $\left(22^{\circ} \mathrm{C}\right)$ with their eyes closed for 45 minutes until imaging began. PET scans were performed in $3 \mathrm{D}$ mode. The CT scan was used for attenuation correction and localization of the ${ }^{18} \mathrm{~F}-\mathrm{FDG}$ uptake sites. Scanning of the head lasted for 10 minutes. The matrix size was $168 \times 168$ and pixel size was $2.04 \times 2.04 \times 1.5 \mathrm{~mm}$ for brain scanning. Hanning filters were used during image reconstruction, giving a transaxial and axial cutoff frequency of 0.5 . The body emission scan was 2 minutes per bed position. Five or 6 bed positions per subject were needed to cover the areas where BAT is usually found. Both PET and CT image sets were reconstructed in transaxial, coronal, and sagittal directions with a section thickness of $3 \mathrm{~mm}$. All studies were performed with the subject's eyes closed in a dimly lit room with minimal auditory stimulation. ${ }^{11}$

One week after the first PET-CT scan, the scans and assessments were repeated in BAT-positive volunteers. In a climate chamber, the subjects rested in a supine position with their eyes closed and were exposed to mild heat $\left(28^{\circ} \mathrm{C}\right)$. After the first 1.25 hours of exposure to heat, the PET tracer ${ }^{18} \mathrm{~F}-\mathrm{FDG}$ was administered intravenously. Then the subjects rested for 45 minutes until imaging began. The total heat exposure time was 2 hours. The brain and body scans were performed as before.

This study followed hospital guidelines and was approved by the ethics committees of Huashan Hospital, Fudan University in Shanghai. All studies were performed after informed consent was obtained.

\section{Data Analysis}

Data on age, sex, height, body weight, FPG, medical history, diagnosis, and average outdoor daily temperature were collected for all subjects. Clinical data analysis was performed by using the SPSS software (Version 17.0; SPSS, Chicago, Illinois), and results were reported as mean \pm SD. Normally distributed continuous variables were compared by means of a paired Student $t$ test for findings of BAT-positive subjects before and after heat exposure and were compared with BATnegative groups by a Student $t$ test. Non-normally distributed continuous variables were compared with the Mann-Whitney $U$ test. All $P$ values presented here are 2-tailed, and values $<.05$ were considered statistically significant.

More than 3 nuclear medicine physicians interpreted the PET-CT images by using open PACS and PET-CT Viewer shareware. Activated BAT was considered present if there were areas of elevated FDG uptake corresponding to the CT attenuation of adipose tissue $(-250$ to $-50 \mathrm{HU}$ ), and it was distinguished from nearby tissue because of distinct patterns compatible with BAT., ${ }^{42}$

PET images of the brain were preprocessed with statistical parametric mapping (SPM2, Wellcome Department of Cognitive Neurology, London, United Kingdom) implemented in Matlab 6.5.1 (MathWorks, Natick, Massachusetts). Scans from each BAT-positive subject were first realigned between pre- and post-heat exposure and were spatially normalized into MNI brain space with linear and nonlinear 3D transformations via the mean image obtained following the realignment. The normalized PET images for BAT-positive subjects and control volunteers were filtered with a Gaussian kernel of $10 \mathrm{~mm}$ (full width at half maximum) in the $\mathrm{x}^{-}, \mathrm{y}^{-}$, and $\mathrm{z}$-axes, respectively. Mean signal-intensity differences over the whole brain were removed by proportional scaling.

To characterize metabolic differences between the BAT-positive and BAT-negative groups, we performed a group comparison by using a 2-sample $t$ test in SPM2 according to the general linear model at each voxel. The effects of heat exposure on regional brain metabolism were also estimated by using a paired $t$ test in the BAT-positive group before and after exposure and a 2-sample $t$ test between the BATpositive group with BAT elimination after exposure and the BATnegative group before exposure. To evaluate significant differences, we used an approach with the peak threshold set at $P=.001$ (uncorrected) for whole brain regions. ${ }^{13,14}$ For hypothesis generation, we reported only clusters that survived an FDR correction at $P<.05$. Significant regions were localized by Talairach Daemon software (http://software.incf.org/software/talairach-daemon) after using a MNI-to-Talairach conversion tool. ${ }^{15}$ SPM maps for increased or decreased metabolism were overlaid on a standard MR imaging brain template.

To quantify regionally specific metabolic changes, we constructed a 4-mm-radius VOI within the image space, centered at the peak voxel of clusters that were significant in the SPM analysis. The VOIs were predefined on the basis of brain regions that were significant in 2-sample $t$ test results of different glucose metabolisms compared with those in BAT-negative controls. We then calculated the relative glucose metabolic rates in pre-exposure and postexposure PET images respectively with ScAnVP software (Version 5.9.1, Center for Neurosciences, The Feinstein Institute for Medical Research, Manhasset, New York). ${ }^{16}$ For all analyses, the significance level was 


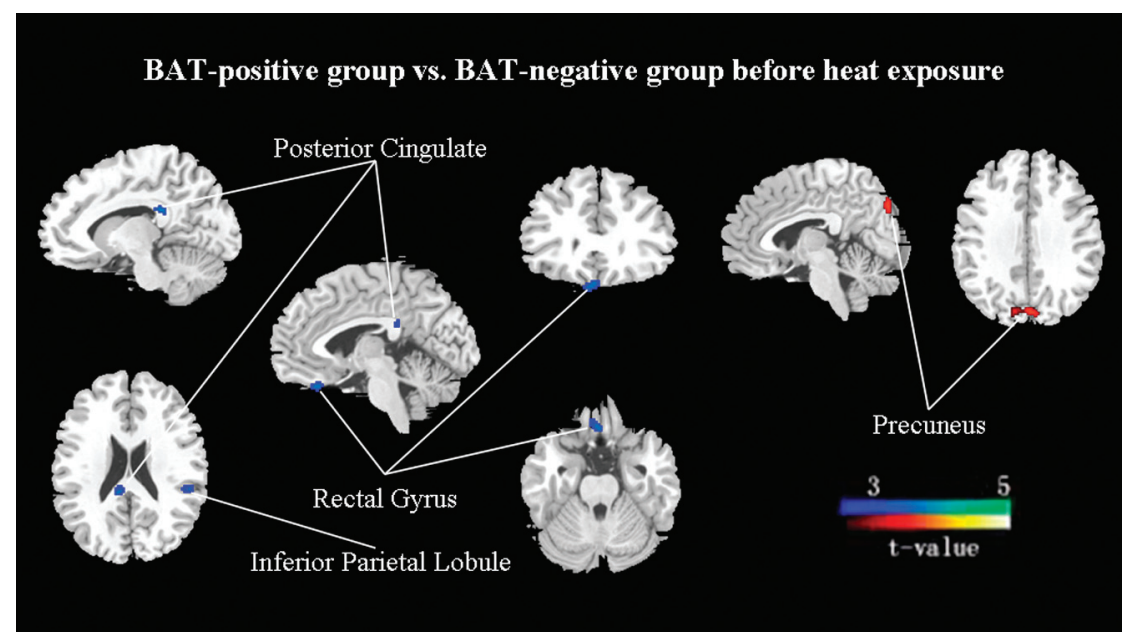

Fig 1. Group differences in regional cerebral activity before heat exposure (FDR correction at $P<.01$ ). The figures are depicted in neurologic orientation. The gray-scale image is a T1 structural MR image that is representative of MNI space. Compared with control subjects, blue areas represent relatively hypometabolic regions and red areas represent relatively hypermetabolic regions in BAT-positive subjects.
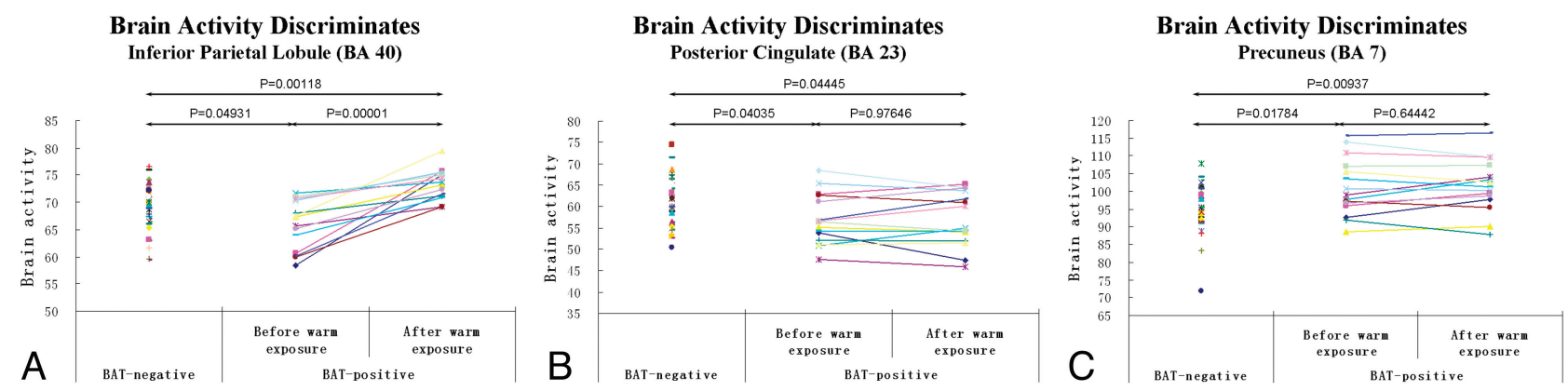

Fig 2. Group discriminates in regional cerebral activity among BAT-negative controls and BAT-positive subjects before and after heat exposure (FDR correction at $P<$.05). The cerebral region of $A$ is the inferior parietal lobule (BA 40), $B$ is the posterior cingulate (BA 23), and $C$ is the precuneus (BA 7).

set at $P<.05$. Statistical analyses were performed by using SPSS software, Version 11.5.

\section{Results}

\section{Clinical Characteristics}

A similar clinical distribution was seen between the BAT-positive and BAT-negative groups. The sex $(P=1.000)$, age $(P=$ $.904)$, BMI $(P=.971)$, FPG $(P=.089)$, and outdoor temperature $(P=.334)$ (On-line Table 1$)$ were all well-matched. Before and after heat exposure, the average outdoor daily temperatures $(8.87 \pm 4.56$ versus $9.07 \pm 6.67, P=.875)$ were also very similar (On-line Table 2 ). There was no detectable BAT activity in the BAT-positive subjects who were retested under heat exposure (On-line Fig 1).

\section{Group Differences in Relative Brain Activity before Heat Exposure}

Compared with BAT-negative controls, the activated BAT subjects showed a significantly decreased metabolism (FDRcorrected $P<.01$ ) in the left inferior parietal lobule (BA 40, $\left.\mathrm{Z}_{\max }=3.50\right)$, right posterior cingulate $\left(\mathrm{BA} 23, \mathrm{Z}_{\max }=3.49\right)$, and right rectal gyrus $\left(\mathrm{BA} 11, \mathrm{Z}_{\max }=3.30\right)$. In contrast, the left precuneus (BA 7, $Z_{\max }=3.59$ ) showed significantly increased metabolism (FDR-corrected $P<.01)$ (On-line Table 3; Figs 1 and 2).

\section{Heat-Exposure-Related Changes in Relative Cerebral Activity}

The second set of PET scans was obtained in all activated BAT subjects 1 week after the first scan. Compared with the images after heat exposure, the baseline findings in the BAT-activated state showed a significant cluster of decreased metabolic activity in the left inferior parietal lobule (BA 40), left postcentral gyrus (BA 5), right subgyrus (BA 6), left inferior frontal gyrus (BA 44), left medial frontal gyrus (BA 6), left middle frontal gyrus (BA 9), right culmen, left insula (BA 13), right lentiform nucleus, and left cingulate gyrus (BA 31) (FDR-corrected $P<$ .05 , On-line Table 4; Figs 2 and 3). No significantly increased metabolism was identified.

However, compared with that in BAT-negative controls, the glucose metabolism after heat exposure was still significantly decreased in the right transverse temporal gyrus (BA 42), right middle temporal gyrus (BA 21), left superior temporal gyrus (BA 22), right cingulate gyrus (BA 31), right posterior cingulate (BA 23), and right parahippocampal gyrus (BA 19) (FDR-corrected $P<.01$, On-line Table 5; Figs 2 and 4). A significant increase was identified in the left inferior pa- 


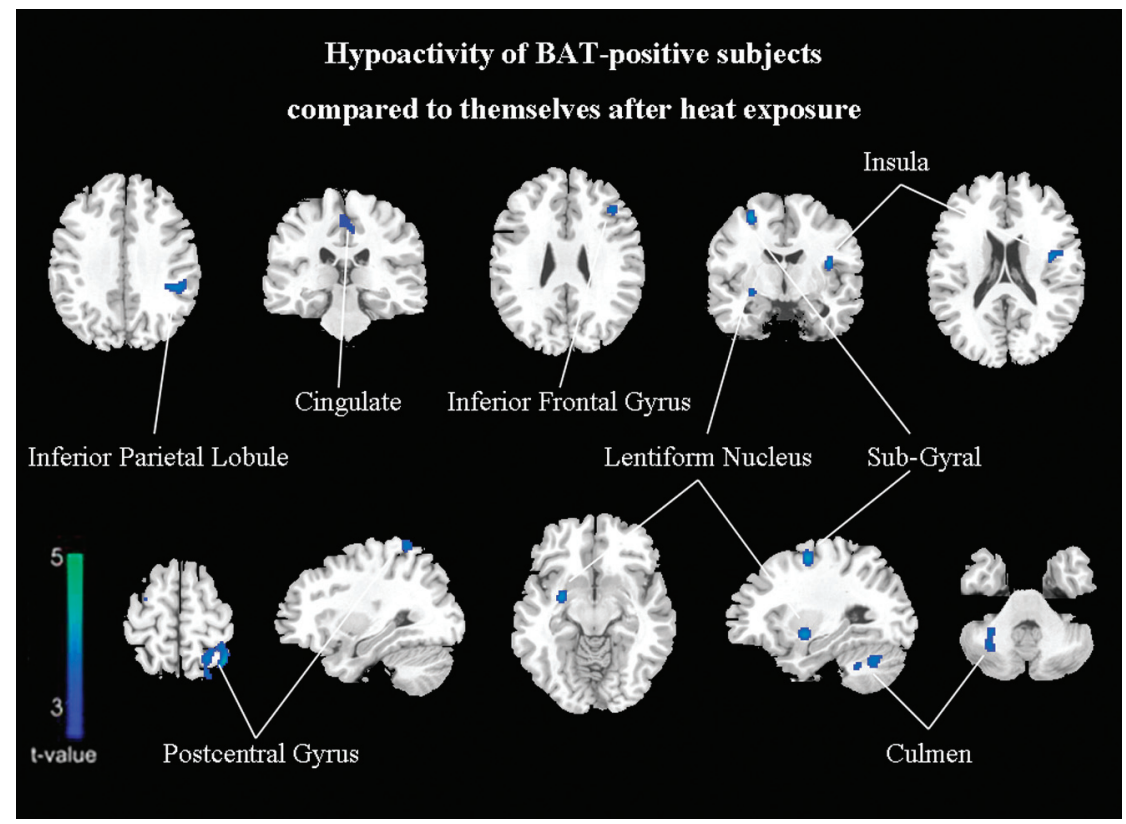

Fig 3. Subjects with activated BAT have relatively hypometabolic regions of decreased FDG uptake compared with themselves after heat exposure (FDR correction at $P<$.05).

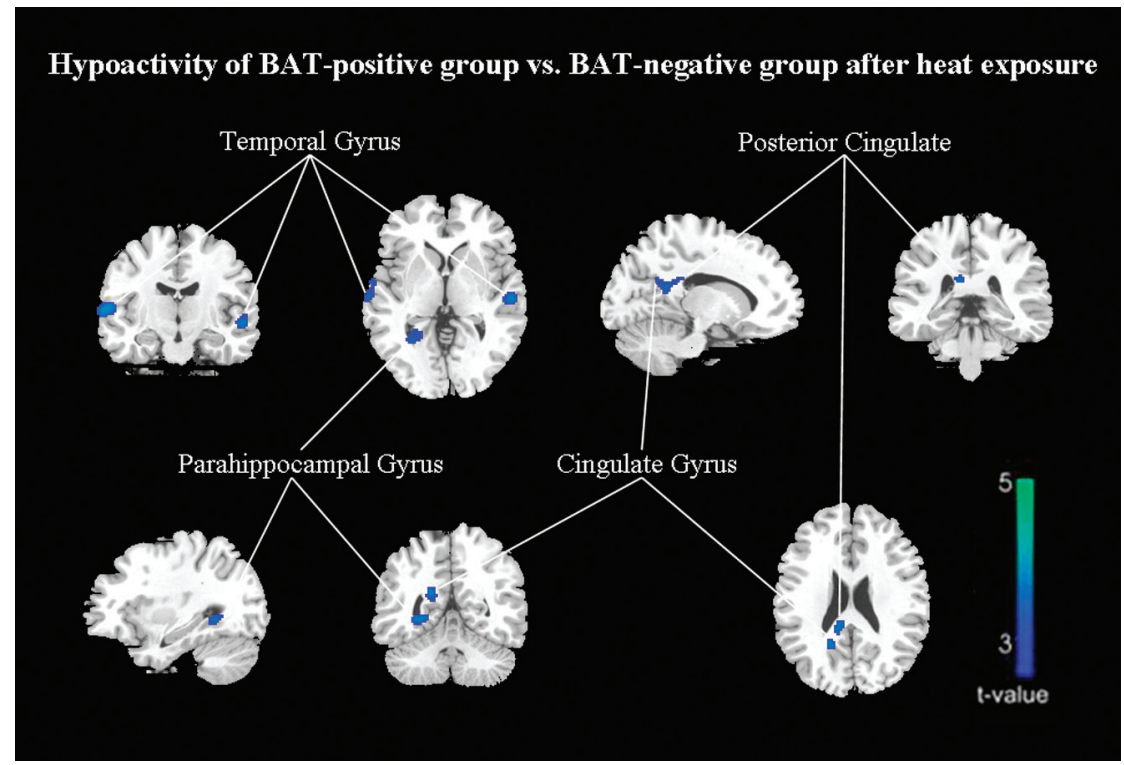

Fig 4. After heat exposure, subjects with inactivated BAT have relatively hypometabolic regions of decreased FDG uptake compared with control subjects (FDR correction at $P<.01$ ).

rietal lobule (BA 40), left precuneus (BA 7), bilateral postcentral gyrus (BA 3), and left cuneus (BA 18) (FDR-corrected $P<$ .01, On-line Table 5; Figs 2 and 5).

\section{Discussion}

This is the first brain functional imaging study about the cerebral metabolic differences following BAT activity change. By using FDG PET/CT, we identified a link between the brain metabolism and BAT activity. In this study, subjects with activated BAT showed significant cerebral metabolic changes following heat exposure, indicating that BAT activity is related to brain metabolism.

The earlier animal studies about the effects of the brain on BAT were focused on the hypothalamic area. This region is involved in several aspects of autonomic regulation, including thermoregulation and energy expenditure. Therefore, it can also influence sympathetically regulated thermogenesis in BAT. It was hypothesized that activation of neurons in the hypothalamus - including dorsomedial neurons, lateral hypothalamus area, ventromedial neurons, medial preoptic area, and raphe pallidus neurons-resulted in sympathoadrenally mediated activation of BAT..$^{7-9,17}$ However, all of these previous studies were in the mammals; which parts of the human brain are related to the activation of BAT is still unknown.

Until 2010, just one study explored the interaction of the brain and the activation of BAT by analyzing differences in brain metabolism between patients with and without activated BAT. These authors used the method of defining regions of interest to examine differences in metabolism between their hypothalami and voxel-based (volumetric pixel) SPM to ana- 


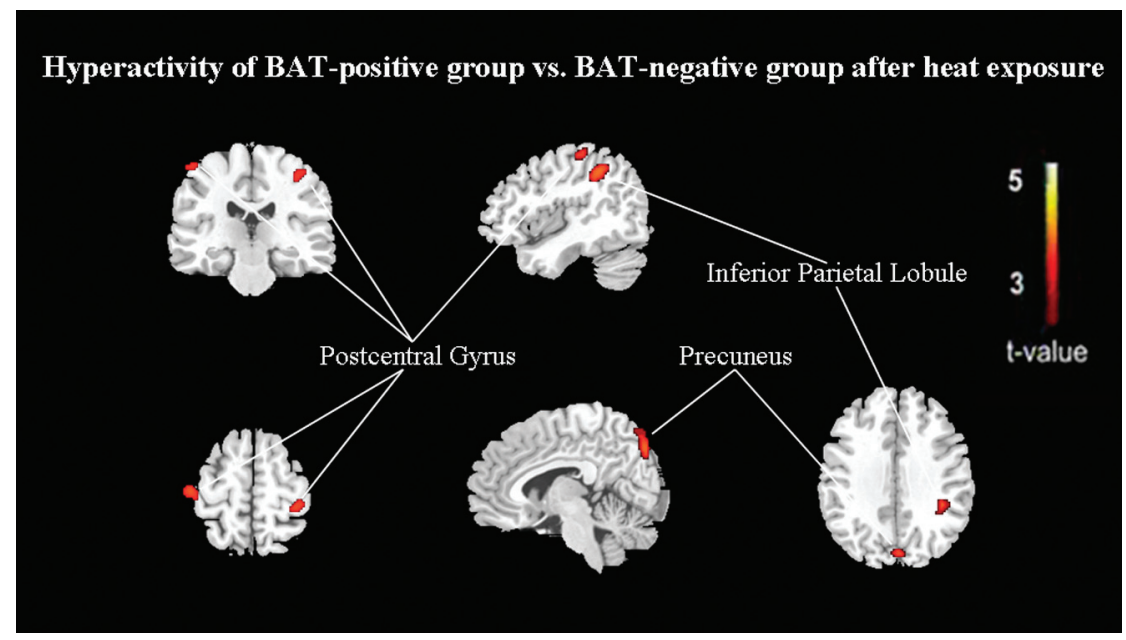

Fig 5. After heat exposure, subjects with inactivated BAT have relatively hypermetabolic regions of increased FDG uptake compared with control subjects (FDR correction at $P<.01$ ).

lyze the whole brain. Compared with the matched controls, the patients with activated-BAT showed an area with significantly increased metabolism in the right inferior parietal lobule (BA 40) and significant hypometabolic areas in the left insula (BA 13) and right cerebellum and there were no metabolic differences in the hypothalamic regions. ${ }^{10}$ This was the first time that the relationship between human brain metabolism and BAT activity by using ${ }^{18}$ F-FDG PET/CT had been explored. However, this study had some limitations. It was retrospective and thus prone to a selection bias. Unobserved differences in the data, such as psychological factors, race, and medical history, could not be controlled and were likely to contribute to the different outcomes.

In contrast to previous imaging studies of BAT activity, our study detected altered metabolism in regions beyond the hypothalamus, parietal lobe, insula, and cerebellum. Compared with BAT-negative controls, the subjects with activated BAT showed altered cerebral metabolism in the inferior parietal lobule, posterior cingulate, rectal gyrus, and precuneus (Online Table 3; Fig 1). These changes may be due to differences in study subjects, research design, and imaging methodology. In previous studies, most animal studies, the PET/CT technique, which is a novel, noninvasive, feasible, and very promising methodology was seldom used. ${ }^{7,8}$ Furthermore, the subjects of the previous retrospective study were patients with cancer, who might be influenced by multiple uncontrollable factors. ${ }^{10}$

Considering the limitations of the retrospective study, we were inclined to select a before-after controlled study, and we believed that the results could be more credible. Studies have shown that keeping the subject warm; administering propranolol, fentanyl, or diazepam; and having subjects follow highfat, low-carbohydrate, and protein-permitted diets before FDG injection can significantly reduce brown fat activity and FDG uptake. ${ }^{18-20}$ Therefore, we selected heat exposure, which was the simplest and had the least impact on the results.

After 2 hours of heat exposure, none of the BAT-positive subjects had detectable BAT activity (On-line Fig 1). In brain analysis, there were many significant brain regional changes following heat exposure (On-line Table 4; Fig 3). These findings implied that temperature stimulation could induce metabolic changes in specific regions of human brain. ${ }^{21}$ The brain regions that had nothing to do with temperature change could be related to BAT activities. To avoid the brain regions that related to temperature change, we compared the BAT-positive group with the BAT-negative group before and after heat exposure. Most interesting, there were 3 brain regions that had significant differences between BAT-positive subjects and BAT-negative controls both before and after heat exposure (On-line Tables 3 and 5; Figs 1, 4, and 5). This finding suggests that these regions were related to the BAT activities between different groups, regardless of temperature changes.

One of the regions was the left inferior parietal lobule (BA 40). Compared with controls, subjects with activated BAT had a significant hypometabolic area in the left inferior parietal lobule (age- and FDR-corrected $P<.01$ ). Following heat exposure, this region showed a significant metabolic increment (FDR-corrected $P<.05$ ). Previous studies revealed that the parietal lobe was dominant for many aspects of somesthetic information processing, including pain, touch, and thermal stimulation..$^{2,23}$ Our findings implied that the left inferior parietal lobule maintained an inhibitory influence on BAT thermogenesis. When exposed to heat, its inhibitory effect decreases the heat production of BAT.

The second important brain region was the right posterior cingulate in the limbic system, which had bidirectional connections with the hypothalamus. The hypothalamus is the primary locus of thermoregulation. The sympathetic nuclei in the hypothalamus modulate nonshivering thermogenesis through the sympathetic activation of uncoupling protein- 1 in BAT. ${ }^{9,24}$ Furthermore, the hypothalamus may both project to the cingulate and receive inhibitory signals from the cingulate via the cingulate hypothalamic circuit. ${ }^{25,26}$ Our study showed that BAT-negative subjects were relatively hypermetabolic in the right posterior cingulate (age- and FDR-corrected $P<$ $.01)$; this finding suggests that the right posterior cingulate might reduce the sympathetic activity of BAT by inhibiting the activity of the hypothalamic nuclei. ${ }^{8}$

The third important brain region was the left precuneus (BA 7). Recent research has shown that the so-called default mode network has become the primary target in recent investigations to link intrinsic activity to cognition and to show how intrinsic signal-intensity changes may be altered in disease. ${ }^{27}$ 
The medial temporal lobe subsystem and the medial prefrontal subsystem converge on important nodes of integration, including the precuneus/posterior cingulate cortex. ${ }^{28,29}$ Additionally, there was support for strong interactions between the precuneus/posterior cingulate cortex and the left inferior parietal lobe. Therefore the role of the precuneus/posterior cingulate cortex in the default mode network is pivotal. ${ }^{27} \mathrm{Com}-$ pared with BAT-negative controls, we found that the activated BAT subjects showed a significantly increased metabolism in the left precuneus (BA 7) before and after heat exposure. This indicated that the precuneus activity could help us distinguish BAT-positive subjects from negative ones.

However, we found no significant differences in the hypothalamus between different BAT activities. The human hypothalamus is a small $\left(4 \mathrm{~cm}^{3}\right)$ but very complex structure; no other brain structure contains so many different small cell groups with different organizations and functions. ${ }^{30}$ The absence of a significant difference in our study suggests that the metabolic change of the hypothalamus is either too subtle to be detected by using our methods or else is attenuated by other metabolic changes caused by numerous hypothalamic functions.

In view of the above-mentioned findings, our results reflect the differences of brain metabolism related to BAT activities. In future research, we may need to explore related factors of the above-mentioned significant regions. On the other hand, the BAT-related central mechanisms in humans may open potential new avenues for the future treatment of obesity and its related disorders.

\section{Conclusions}

On the basis of our before-after controlled study, we found that local brain metabolism was related to BAT activity. The glucose metabolism in the left inferior parietal lobule, the right posterior cingulate, and the left precuneus is important for controlling BAT thermogenesis within the central pathways regardless of temperature changes. Our findings support the hypothesis that the BAT-related central mechanism may be a target for pharmacologic interventions that modulate energy expenditure to treat obesity and obesity-related diseases.

\section{Acknowledgments}

We sincerely thank the volunteers who participated.

\section{References}

1. Cannon B, Nedergaard J. Brown adipose tissue: function and physiological significance. Physiol Rev 2004;84:277-359

2. Lowell BB, Spiegelman BM. Towards a molecular understanding of adaptive thermogenesis. Nature 2000;404:652-60

3. Nedergaard J, Bengtsson T, Cannon B. Unexpected evidence for active brown adipose tissue in adult humans. Am J Physiol Endocrinol Metab 2007;293: E444-52

4. Cypess AM, Lehman S, Williams G, et al. Identification and importance of brown adipose tissue in adult humans. $N$ Engl J Med 2009;360:1509-17

5. Virtanen KA, Lidell ME, Orava J, et al. Functional brown adipose tissue in healthy adults. $N$ Engl J Med 2009;360:1518-25

6. Seydoux J, Girardier L. Control of brown fat thermogenesis by the sympathetic nervous system. Experientia Suppl 1978;32:153-67

7. Niijima A, Rohner-Jeanrenaud F, Jeanrenaud B. Role of ventromedial hypothalamus on sympathetic efferents of brown adipose tissue. Am J Physiol 1984; 247(4 pt 2):R650-54

8. Cerri M, Morrison SF. Activation of lateral hypothalamic neurons stimulates brown adipose tissue thermogenesis. Neuroscience 2005; 135:627-38

9. Morrison SF. Central pathways controlling brown adipose tissue thermogenesis. News Physiol Sci 2004;19:67-74

10. Huang YC, Hsu CC, Huang $\mathrm{P}$, et al. The changes in brain metabolism in people with activated brown adipose tissue: a PET study. Neuroimage 2010;54:142-47

11. Wang J, Ma Y, Huang Z, et al. Modulation of metabolic brain function by bilateral subthalamic nucleus stimulation in the treatment of Parkinson's disease. J Neurol 2009;257:72-78

12. Cohade C, Osman M, Pannu HK, et al. Uptake in supraclavicular area fat (“USA-Fat"): description on 18F-FDG PET/CT. J Nucl Med 2003;44:170-76

13. Saxena S, Brody AL, Ho ML, et al. Differential brain metabolic predictors of response to paroxetine in obsessive-compulsive disorder versus major depression. Am J Psychiatry 2003;160:522-32

14. Smith GS, Kramer E, Hermann C, et al. Serotonin modulation of cerebral glucose metabolism in depressed older adults. Biol Psychiatry 2009;66:259-66

15. Lancaster JL, Woldorff MG, Parsons LM, et al. Automated Talairach atlas labels for functional brain mapping. Hum Brain Mapp 2000;10:120-31

16. Huang $C$, Tang $C$, Feigin $A$, et al. Changes in network activity with the progression of Parkinson's disease. Brain 2007;130:1834-46

17. Zaretskaia MV, Zaretsky DV, Shekhar A, et al. Chemical stimulation of the dorsomedial hypothalamus evokes non-shivering thermogenesis in anesthetized rats. Brain Res 2002;928:113-25

18. Basu S, Alavi A. Optimizing interventions for preventing uptake in the brown adipose tissue in FDG-PET. Eur J Nucl Med Mol Imaging 2008;35:1421-23

19. Christensen CR, Clark PB, Morton KA. Reversal of hypermetabolic brown adipose tissue in F-18 FDG PET imaging. Clin Nucl Med 2006;31:193-96

20. Williams G, Kolodny GM. Method for decreasing uptake of 18F-FDG by hypermetabolic brown adipose tissue on PET. AJR Am J Roentgenol 2008;190:1406-09

21. Fechir M, Klega A, Buchholz HG, et al. Cortical control of thermoregulatory sympathetic activation. Eur J Neurosci 2010;31:2101-11

22. Peyron R, Garcia-Larrea L, Gregoire MC, et al. Allodynia after lateral-medullary (Wallenberg) infarct: a PET study. Brain 1998;121(pt 2):345-56

23. Tracey I, Becerra L, Chang I, et al. Noxious hot and cold stimulation produce common patterns of brain activation in humans: a functional magnetic resonance imaging study. Neurosci Lett 2000;288:159-62

24. Collins S, Surwit RS. The beta-adrenergic receptors and the control of adipose tissue metabolism and thermogenesis. Recent Prog Horm Res 2001;56:309-28

25. Samuels BC, Zaretsky DV, DiMicco JA. Dorsomedial hypothalamic sites where disinhibition evokes tachycardia correlate with location of raphe-projecting neurons. Am J Physiol Regul Integr Comp Physiol 2004;287:R472-78. Epub 2004 Apr 8.

26. Vogt BA, Vogt L, Laureys S. Cytology and functionally correlated circuits of human posterior cingulate areas. Neuroimage 2006;29:452-66

27. Fransson $P$, Marrelec $G$. The precuneus/posterior cingulate cortex plays a pivotal role in the default mode network: evidence from a partial correlation network analysis. Neuroimage 2008;42:1178-84

28. Gusnard DA, Akbudak E, Shulman GL, et al. Medial prefrontal cortex and self-referential mental activity: relation to a default mode of brain function. Proc Natl Acad Sci U S A 2001;98:4259-64

29. Buckner RL, Andrews-Hanna JR, Schacter DL. The brain's default network: anatomy, function, and relevance to disease. Ann N Y Acad Sci U S A 2008;1124:1-38

30. Hofman MA, Swaab DF. The human hypothalamus: comparative morphometry and photoperiodic influences. Prog Brain Res 1992;93:133-47, discussion $148-49$ 